\title{
Molecular Evolution of the Sex Peptide Network in Drosophila
}

\author{
Meaghan K. McGeary ${ }^{1,2}$ and Geoffrey D. Findlay ${ }^{1, *}$ \\ ${ }^{1}$ Department of Biology, College of the Holy Cross, Worcester, MA 01610 \\ ${ }^{2}$ Department of Pathology, Yale School of Medicine, New Haven, CT 06520 \\ *Correspondence to: gfindlay@holycross.edu, 508-793-2655 (office), 508-793-2656 (fax)
}

Short title: Drosophila sex peptide network evolution 


\section{Abstract}

Successful reproduction depends on interactions between numerous proteins beyond

3 those involved directly in gamete fusion. While such reproductive proteins evolve in response

4 to sexual selection pressures, how networks of interacting proteins arise and evolve as

5 reproductive phenotypes change remains an open question. Here, we investigated the molecular

6 evolution of the "sex peptide network" of Drosophila melanogaster, a functionally well-

7 characterized reproductive protein network. In this species, the peptide hormone sex peptide

8 (SP) and its interacting proteins cause major changes in female physiology and behavior after

9 mating. In contrast, females of more distantly related Drosophila species do not respond to SP.

10 In spite of these phenotypic differences, we detected orthologs of all network proteins across 22

11 diverse Drosophila species and found evidence that most orthologs likely function in

12 reproduction throughout the genus. Within SP-responsive species, we detected the recurrent,

13 adaptive evolution of several network proteins, consistent with sexual selection acting to

14 continually refine network function. We also found some evidence for adaptive evolution of

15 several proteins along two specific phylogenetic lineages that correspond with increased

16 expression of the SP receptor in female reproductive tracts or increased sperm length,

17 respectively. Finally, we used gene expression profiling to examine the likely degree of

18 functional conservation of the paralogs of an SP network protein that arose via gene duplication.

19 Our results suggest a dynamic history for the SP network in which network members arose

20 before the onset of robust SP-mediated responses and then were shaped by both purifying and

21 positive selection.

23 Keywords: sex peptide, Drosophila, seminal fluid, sexual selection, molecular evolution 


\section{Introduction}

Successful reproduction requires the fusion of egg and sperm cells, yet this fusion is often

27 facilitated by proteins that are not part of the gametes. For example, non-gametic reproductive

28 proteins provided in male seminal fluid or produced in the female reproductive tract can facilitate

29 sperm motility, induce or manage sperm storage or cause changes to female reproductive

30 physiology (Wilburn \& Swanson, 2016, Schnakenberg et al., 2011). While proteomic and

31 comparative genomic methods have enabled the identification of hundreds of gametic and non-

32 gametic reproductive proteins across diverse taxa (reviewed in McDonough et al., 2016),

33 understanding how these proteins interact, and how such interactions evolve, remain areas of

34 active research.

35 Some of the best-characterized reproductive protein interactions occur in the "sex peptide

36 network" of Drosophila melanogaster that regulates female post-mating behavior and

37 physiology. The network centers on the sex peptide (SP), a short peptide hormone transferred

38 from males to females as a non-gametic component of seminal fluid (Chen et al., 1988). The

39 presence of SP in the female reproductive tract stimulates egg production (Soller et al., 1999),

40 reduces receptivity to remating (Liu \& Kubli, 2003, Chapman et al., 2003), facilitates the release

41 of sperm from storage prior to fertilization (Avila et al., 2010), and affects numerous other

42 female behaviors, including feeding, defecation and sleep (Carvalho et al., 2006, Apger-

43 McGlaughon \& Wolfner, 2013, Isaac et al., 2010). SP-mediated effects on females persist for

44 several days after mating because SP binds to sperm, which become stored in specialized storage

45 organs in the female tract (Peng et al., 2005). SP is then gradually cleaved from sperm and

46 released from the storage organs into the female tract, where it interacts with the sex peptide

47 receptor (SPR), a G-protein coupled receptor that is expressed in a subset of neurons innervating 
48 the uterus (Yapici et al., 2008, Hasemeyer et al., 2009, Yang et al., 2009). This gradual "dosing”

49 of SP causes the persistence of the hormone's effects on female behavior and physiology. SPR

50 signaling is also required for the efficient release of sperm from the storage organs (Avila et al.,

$512015)$

52 While the molecule(s) on sperm to which SP binds remain unknown, RNAi screens have

53 identified several additional male seminal fluid proteins and female reproductive tract proteins

54 required for robust SP responses (Ravi Ram \& Wolfner, 2007, Ravi Ram \& Wolfner, 2009,

55 LaFlamme et al., 2012, Findlay et al., 2014, Singh et al., 2018). Together with SP and SPR,

56 these proteins comprise the SP network. The male-derived proteins include: predicted C-type

57 lectins CG1652 and CG1656; predicted proteases/protease homologs CG9997, seminase,

58 aquarius and intrepid; and, predicted cysteine-rich secretory proteins CG17575 and antares. The

59 female-derived proteins include fra mauro (a predicted metallopeptidase), Esp (a predicted anion

60 transporter) and hadley (which lacks identifiable protein domains). The male-derived proteins

61 act interdependently to facilitate SP binding to sperm (Ravi Ram \& Wolfner, 2009, Findlay et

62 al., 2014, Singh et al., 2018), while the female-derived proteins act downstream of SP binding to

63 sperm, potentially by facilitating SP-SPR signaling (Findlay et al., 2014). Other genes expressed

64 in the secondary cells of the male accessory gland are also required for SP-mediated responses,

65 though it remains unclear whether these genes encode proteins that interact directly with the

66 network proteins described above (Sitnik et al., 2016).

67 SP's functions and interactions have been well characterized in D. melanogaster, but

68 comparative genomic and functional studies have shown that the SP response is not conserved

69 throughout the Drosophila genus. Tsuda et al. (2015) found that only species of the

70 melanogaster group of Drosophila (Fig. 1) show changes in female remating receptivity and egg 
71 production upon injection with synthetic SP, even though SP and SPR orthologs can be detected

72 outside of this group. Furthermore, by incubating GFP-labeled SP with female reproductive

73 tracts from progressively more divergent species, Tsuda et al. (2015) discovered that SP could

74 bind to the female tract only in melanogaster group species. This observation suggested that

75 robust expression of SPR in the female tract evolved on the phylogenetic lineage leading to the

76 melanogaster group, which the authors tested by comparing $S P R$ gene expression between in-

77 group and out-group species (Tsuda et al., 2015). Consistent with D. melanogaster expression

78 patterns (Yapici et al., 2008), they found that $S P R$ was expressed in non-reproductive areas in

79 both sexes of all species examined. However, its expression in the female reproductive tract was

80 largely limited to the melanogaster group. (The only outgroup species that showed expression in

81 this location was D. virilis, but conspecific GFP-labeled SP did not bind to female reproductive

82 tracts in this species.) Intriguingly, the SP ortholog from D. pseudoobscura (a non-melanogaster

83 group species) is expressed in D. pseudoobscura male reproductive tracts (Yang et al., 2018) and

84 can trigger SP-mediated responses when injected into D. melanogaster females, but not when

85 injected into conspecifics (Tsuda et al., 2015). This result suggests that the SP protein might have

86 evolved the potential to affect female post-mating behavior before the emergence of the

87 melanogaster group, but this function was not fully realized until the subsequent evolution of

88 SPR expression in the female reproductive tract (and, perhaps, within specific neurons in the

89 tract) (Hasemeyer et al., 2009, Yang et al., 2009, Yapici et al., 2008, Rezaval et al., 2012). It is

90 also possible that the transition to high levels of SPR expression in the female reproductive tract

91 created or intensified an evolutionary selective pressure to bind higher levels of SP to stored

92 sperm. 
While Drosophila species differ in reproductive traits for many reasons (Markow \&

94 O'Grady, 2005), some of these differences relate directly to the SP network and could thus be

95 causes or consequences of SP network evolution. For example, a change in sperm length may

96 affect the amount of SP that can bind, and other structural changes to sperm could affect the

97 binding of SP and other network proteins that interact with sperm, such as CG1652 and CG1656

98 (Ravi Ram \& Wolfner, 2009, Singh et al., 2018). The amount of SP bound to sperm, the rate and

99 efficacy of its release (Peng et al., 2005), and its ability to bind SPR (Yapici et al., 2008) could

100 affect re-mating rates, while the structure of female sperm storage organs could affect the ability

101 of the network proteins to bind SP to sperm or modulate SP's interaction with SPR. Changes in

102 these traits - sperm length, female remating rate, and female sperm storage structures - have

103 been well documented in the literature (Alpern et al., 2019, Joly \& Bressac, 1994, Markow,

104 1996, Markow \& O'Grady, 2005, Pitnick et al., 1999, Snook et al., 1994, Snook, 1995, Singh et

105 al., 2002), and we summarize them and infer their timing in Figure 1. Of particular relevance to

106 this study, the phenotypic and phylogenetic data are consistent with SPR evolving to be

107 expressed in female reproductive tracts along the lineage leading to the melanogaster group of

108 flies (Tsuda et al., 2015; branch 11) and an increase in sperm length on the lineage leading to $D$.

109 ananassae and D. bipectinata (Joly \& Bressac, 1994, Markow, 1996, Pitnick et al., 1999; branch

$11015)$.

In light of the differences between species in reproductive phenotypes, we used

112 comparative genomics and molecular evolutionary analysis to gain insights into the evolution of

113 the SP network. While robust, long-lasting changes in female behavior and physiology due to

114 SP are found only in the melanogaster group of Drosophila, we identified orthologs of each SP

115 network protein in numerous outgroup species and verified their expression in the male 
116 reproductive system in two such species from published data (Yang et al., 2018, Kelleher et al., 117 2009). Using PAML (Yang, 2007) we determined that recurrent positive selection has acted on

118 specific sites in several of these proteins. We also detected marginal evidence that positive

119 selection has acted on certain network proteins on key phylogenetic lineages corresponding with

120 major changes in SP-related phenotypes. Finally, we used RT-PCR to investigate whether any

121 gross-scale changes in gene expression occurred surrounding the gene duplication event that

122 gave rise to one of the SP network proteins, seminase. Taken together, our results suggest that

123 the members of the SP network had the potential to influence reproductive success before the

124 onset of SP/SPR-mediated responses in the reproductive tract of mated females of the

125 melanogaster group of species. However, additional adaptive changes in these proteins occurred

126 concurrent with, and subsequent to, these critical changes in the fly reproductive system. These

127 results underscore the strength of sexual selection acting in Drosophila and illustrate potential

128 molecular changes that occur in the face of such selection. 
Methods

Identification of SP network proteins across Drosophila species

We obtained the protein sequence for each SP network protein in D. melanogaster from

132 FlyBase. For species for which protein annotations were available on FlyBase (Drosophila 12

133 Genomes Consortium, 2007), we obtained orthologous protein-coding DNA sequences using the

134 FlyBase Orthologs feature. These species included Drosophila simulans, sechellia, yakuba, erecta, ananassae, pseudoobscura, persimillis, willistoni, mojavensis, virilis and grimshawi. For species with sequenced genomes that lacked FlyBase protein annotations (Chen et al., 2014), we manually searched for gene orthologs using tBLASTn and the D. melanogaster protein sequence as the query. These species include Drosophila ficusphila, eugracilis, takahashii, elegans,

139 rhopoloa, kikkawai, bipectinata, miranda and albomicans. For genes expected to have introns

140 based on the D. melanogaster gene structure, we looked in the unannotated species for the

141 approximate location of the D. melanogaster intron, and used known intron border consensus

142 sequences and six-frame translation, implemented in EMBOSS SixPack (Madeira et al., 2019),

143 to identify predicted intron borders and remove intronic sequences prior to the analyses below.

144 To study the gene duplication events that gave rise to seminase and its tandem gene

145 duplicates (CG10587 and CG11037 in D. melanogaster), we identified the genes flanking these

146 three genes and used them to identify the syntenic region of the other Drosophila genomes. We

147 assumed conservation of gene order within this syntenic region in assigning orthologs for this

148 gene family (Figure S1).

For all putative orthologs identified by bioinformatic methods, we verified that the ortholog was the reciprocal best BLAST hit to the expected SP network member of $D$.

151 melanogaster. Inferred orthologs with a high degree of similarity, successful reciprocal best hits, 
152 and a sequence that could be translated conceptually to produce a polypeptide without premature

153 stops, were retained for study. In cases of duplicate genes (seminase, CG1652 and CG1656), we

154 also used gene order and synteny to confirm correct ortholog identification.

155

Sequence alignment

For each SP network protein, we used MUSCLE as implemented in MEGA 6.06 (Tamura et al., 2013) to align amino acid sequences, then visually checked and edited each alignment for accuracy. Amino acid alignments were then back-translated in MEGA to obtain the cDNA

160 alignment.

\section{Phylogenetic analysis}

164 concatenated the amino acid alignments of all SP network proteins within each of the 22 species.

165 We used PROML in Phylip (Felsenstein, 2005) to infer an unrooted maximum-likelihood

166 phylogeny (with random input order, slow analysis, and all other default parameters). Gaps in the

167 alignment were used in cases in which a protein was not present in a particular species. The

168 resulting phylogeny matched published Drosophila phylogenies, except for $D$. virilis and $D$.

169 mojavensis (Drosophila 12 Genomes et al., 2007, Markow \& O'Grady, 2005, Seetharam \&

170 Stuart, 2013). We then used this consensus tree for the PAML analyses, with species removed

171 on a gene-by-gene basis as described below. 
Because recombination within a gene sequence can impact the results of analyses to

175 detect selection, we first used GARD with default parameters as implemented in DataMonkey

1762.0 to check for evidence of recombination within each gene (Kosakovsky Pond et al., 2006,

177 Weaver et al., 2018). Genes were partitioned at breakpoints evaluated as significant by the

178 Kishino-Hasegawa test (p-value $<0.05$ for both LH and RH; Table S2), and PAML was run on

179 each gene segment separately. We performed PAML analyses on sequence alignments spanning

180 two different ranges in the Drosophila phylogeny: the branch and branch-sites tests (see below)

181 were run on species from the entire genus, while the sites test (see below) was run on species

182 from only the melanogaster group. Thus, we generated a set of recombination breakpoints for

183 each set of species (i.e., all examined members of the genus or members of the melanogaster

184 group; Table S2). For each species set, six SP network genes showed evidence of

185 recombination, but the genes that showed recombination differed between the two sets of

186 species.

PAML analyses

For each protein, we used codeml of the PAML package to perform evolutionary

191 each protein's evolution across the phylogeny, we utilized the PAML branch test, which uses a

192 likelihood ratio test (LRT) to compare the "free ratio" model, allowing for different $\omega$ values for

193 each branch, with model M0, which estimates a single $\omega$ for the whole phylogeny (Yang, 1998).

194 For these tests, and for the branch-sites tests below, we used the consensus tree described above

195 that covered the entire Drosophila phylogeny, but manually removed from it any species for

196 which: a) an ortholog could not be identified, or b) an ortholog was identified, but it could not be 
197 confidently aligned due to ambiguity over an intron position or the end of the protein-coding 198 region. Table S1 shows the set of species used for the molecular evolutionary analyses for each 199 gene.

200 To test whether a subset of sites in a protein had evolved under recurrent positive

201 selection, we used LRTs to compare an evolutionary model (M8) that allows a class of sites to

202 have $\omega>1$ to models M7 and M8a, which allow only purifying selection or neutral evolution

203 (Swanson et al., 2003, Yang et al., 2000). If model M8 can explain the observed sequence data

204 significantly better than models M7 and M8a, as assessed by a LRT, then positive selection 205 acting on a subset of sites can be inferred. For proteins for which model M8 was significantly

206 preferred to models M7 and M8a, we used the Bayes Empirical Bayes (BEB) approach to

207 identify at the 0.9 confidence level the specific residues that have evolved adaptively. These

208 comparisons were done only for species within the melanogaster group due to the possibility of

209 synonymous site saturation if more divergent species were included. (Such saturation occurs

210 when the rate of substitutions at synonymous sites, $d_{\mathrm{s}}$, approaches 1 , since it then becomes

211 impossible to distinguish whether one or multiple mutations have occurred at these sites. This,

212 in turn, reduces the accuracy of estimates of $d_{\mathrm{N}} / d_{\mathrm{s}}$.) To check for convergence in the 'free-ratio'

213 and the sites models, we ran codeml twice with the initial omega set at 0.4 and 2, respectively.

214 Finally, we performed the branch-sites test for positive selection (Zhang et al., 2005) to

215 identify classes of sites that had evolved adaptively along either of two specific lineages in the

216 phylogeny that we identified a priori because they represent likely evolutionary transitions in

217 key SP-related traits. First, we tested for sites under selection on the branch leading to the

218 melanogaster group of species (Fig. 1, branch 11), since this branch corresponds with the

219 inferred timing of when the SP receptor became expressed in the female reproductive tract and, 
220 consequently, when females became sensitive to the non-receptivity effect caused by SP (Tsuda

221 et al., 2015). Second, we tested for sites under selection in the lineage that leads to and separates

222 D. ananassae and D. bipectinata from the rest of the melanogaster group species (Fig. 1, branch

223 15), because these species are known to have somewhat longer sperm (Joly \& Bressac, 1994,

224 Markow, 1996, Pitnick et al., 1999). Although we inferred other important evolutionary

225 transitions in reproductive traits on the broader Drosophila phylogeny (Fig. 1), we limited our

226 branch-sites analyses to these two lineages because of the greater number of available sequenced

227 species in the melanogaster group.

228 In the branch-sites test, we used a LRT to compare a null model allowing for only

229 purifying and neutral selection on the focal branch with an alternative model allowing for a class

230 of sites to evolve under positive selection (Yang, 2007, Yang \& Dos Reis, 2011, Zhang et al.,

231 2005). Recently, Venkat et al. (2018) found that this branch-sites test can have a high rate of

232 false positives driven by multinucleotide mutations within codons (i.e., mutations at adjacent

233 sites). To control for this issue, we implemented the tests in the Venkat model, a version of

234 PAML developed by these authors that runs the analysis after masking these sites. PAML

235 analyses were implemented using custom batch scripts for GNU parallel (Tange, 2018) and

236 PAML version 4.8a or HyPhy version 2.5.1 (in the case of the Venkat model).

Identification of seminase orthologs and paralogs

We identified the predicted amino acid sequences for orthologs of seminase, CG11037

240 and CG10587 in Drosophila species using the methods described above. To confirm that calls of

241 orthology for seminase and its paralogs were accurate, we used Phylip's PROML program

242 (Felsenstein, 2005) to infer a maximum-likelihood rooted tree (using the single copies in $D$. 
243 pseudoobscura and D. persimillis as the outgroup, and default PROML parameters). This was

244 consistent with the orthology assignments made using conserved gene order, except for $D$.

245 ananassae and D. bipectinata, which are likely confounded by their long branch length.

247 Evaluation of gene expression

248 D. melanogaster, D. yakuba, D. ficusphila, D. bipectinata, D. annanassae, D.

249 pseudoobscura and D. willistoni were raised in the lab as in Tsuda et al. (2015). We $\mathrm{CO}_{2}$ -

250 anesthetized 9-day-old flies of each species, separated them by sex, homogenized male or female

251 whole flies in TRIzol reagent, and purified RNA from samples and synthesized cDNA as

252 previously described (Gubala et al., 2017). We then used species-specific primers to amplify

253 seminase, CG1 1037 or CG10587, with the non-reproductive, housekeeping gene, ribosomal

254 protein $L 32(R p L 32)$ as a control that should be expressed equally in both sexes. Genomic DNA

255 was used as a positive control for PCR reactions, and water was used in place of template in

256 negative control reactions. 


\section{Results and Discussion}

SP network proteins are present in species outside of the melanogaster group

While SP orthologs have been found in species outside of the melanogaster group, only

260 females of species within this group appear to show large-scale SP-mediated reproductive

261 responses (Tsuda et al., 2015). One likely factor for this change is the evolution of SPR

262 expression in the female reproductive tract in the last common ancestor of the melanogaster

263 group (Tsuda et al., 2015). This evolutionary history raises the question of whether the

264 remaining members of the SP network - all of which are critical for SP responses in $D$.

265 melanogaster - are present outside of the group. We addressed this question bioinformatically

266 by searching for intact orthologs across 22 Drosophila species with sequenced genomes.

Figure 2 shows that all SP network protein-coding gene orthologs are detectable in a

268 large majority of the species surveyed, including those outside of the melanogaster group. For

269 example, we found all currently known network proteins in D. pseudoobscura and D. willistoni,

270 and all but one ortholog in $D$. virilis. To assess whether these orthologs were likely to function

271 in reproduction outside of the melanogaster group, we examined publicly availably RNAseq data

272 from male reproductive tracts in D. pseudoobscura (Yang et al., 2018) and proteomic data from

273 male accessory glands in D. mojavensis (Kelleher et al., 2009). Transcripts of orthologs of male-

274 derived network proteins were consistently enriched in (or entirely specific to) samples from

275 whole males, male testes, and male carcasses in D. pseudoobscura, while showing either no or

276 low expression in females or in male heads (Fig. S1). Expression enrichment in whole males and

277 male carcasses is consistent with expectations for reproductive proteins produced in the male

278 accessory gland. Some of these proteins may also be expressed in the testes, or the "testis"

279 expression could be due to contamination of testis dissections with accessory gland tissue. The 
genes encoding female-derived proteins showed broader expression patterns (Fig. S1), including in whole females and whole males, but this pattern is consistent with their D. melanogaster orthologs, the expression of which is not limited to the female reproductive system (Brown et al., 2014). Predicted orthologs of the male-derived network proteins CG1652, CG1656, CG9997, CG17575, seminase, aquarius and antares were also identified in a proteomic analysis of the $D$. mojavensis accessory gland (Kelleher et al., 2009). Subsequent work showed that males of this species transfer transcripts of the antares ortholog to females during mating (Bono et al., 2011). Thus, RNAseq and proteomic data from two outgroup species are consistent with many SP network proteins functioning in reproduction outside of the melanogaster group. that impact their evolutionary trajectories. For example, SPR is expressed in both sexes outside

291 of the reproductive tract (Tsuda et al., 2015, Yapici et al., 2008), and myoinhibitory peptides

292 (MIPs) are known ligands in addition to SP (Kim et al., 2010, Poels et al., 2010, Yamanaka et al., 2010). SPR-MIP interactions outside of the reproductive tract affect sleep patterns in both sexes

294 and remating propensity in females (Jang et al., 2017, Oh et al., 2014). Such interactions, in

295 addition to the sexual selective pressures exerted by SP network-mediated interactions and 296 reproductive phenotypes, have likely contributed to the evolution of SP network proteins in

297 various Drosophila lineages. While most male-derived network proteins appear to have male-

298 specific or heavily male-biased expression (in species for which expression data are available),

299 the female-derived proteins show broader expression patterns. Understanding these proteins'

300 non-reproductive functions will shed additional light on evolutionary forces that may have

301 shaped them. 
SP network proteins demonstrate evolutionary rate heterogeneity across the Drosophila

phylogeny

Because recombination within a gene can cause false positive results in the PAML analyses, we first analyzed each set of orthologs using GARD (Kosakovsky Pond et al., 2006) to identify high-confidence recombination sites, which were detected for six of the proteins (Table S2). These six proteins were thus split into segments corresponding to the regions between recombination breakpoints, which we analyzed independently.

311 PAML model M0 to estimate for each gene a single $d_{\mathrm{N}} / d_{\mathrm{S}}$ ratio $(\omega)$ across all species for either

312 the full-length protein-coding sequence or for each segment identified by the GARD analysis.

313 We then performed the branch test (Yang, 1998) to assess whether $\omega$ varied significantly across

314 different branches of the phylogeny. Most network proteins (and segments of proteins) had full-

315 length $\omega$ estimates around 0.2 across the full-genus tree (Table S3). While these rates are

316 generally indicative of purifying selection, they are nonetheless faster than those observed for

317 most proteins in D. melanogaster (Drosophila 12 Genomes Consortium, 2007), consistent with

318 the general trend for reproductive proteins (Wilburn \& Swanson, 2016). Three proteins showed notably slower evolutionary rates: CG17575 (most of protein found in segments with $\omega<0.1$ ), a male-expressed cysteine-rich secretory protein required for binding of SP to sperm (Ravi Ram \&

321 Wolfner, 2009); Esp ( $\omega=0.03)$, a female-expressed, predicted sulfate membrane transporter also

322 required for long-term fertility (Findlay et al., 2014); and SPR (most of the protein contained in a

323 segment with $\omega=0.05$ ), the female-expressed G-protein coupled receptor for SP required for

324 female post-mating changes including egg-laying, resistance to remating and release of sperm

325 from sperm-storage organs (Avila et al., 2015, Yapici et al., 2008). While these proteins' slow 
rates of evolution could indicate that they play highly conserved roles in reproduction, it is also

327 possible that they have evolved adaptively at only a few sites or on a few lineages (see below).

332 test of positive selection acting on specific branches, the results indicate that the evolutionary

333 rates of most SP network proteins have varied significantly across their evolutionary histories.

334 Indeed, several network proteins had branch-specific $\omega$ estimates $>1$ in some of the more

335 ancestral branches of the tree, highlighting the potential for more detailed studies of these

336 proteins' evolutionary histories as the genomes of more divergent Drosophila species become

337 available. In contrast, the constant, slow rate of evolution for intrepid implies that this protein

338 has likely played a conserved and important role since the origin of the genus. Intrepid has

339 undergone less functional characterization than other male-expressed male network proteins, so

340 we cannot speculate further about its specific role(s) in reproduction.

342 Several SP network proteins have undergone recurrent positive selection at specific sites since

343 the evolution of SPR expression in female reproductive tracts

To determine the extent to which positive selection has shaped the evolution of the SP network proteins, we used the PAML sites test to ask whether any protein had a particular subset

346 of sites that had undergone recurrent positive selection. Because of the likelihood of

347 synonymous site saturation over longer phylogenetic distances, we limited the sequences used in

348 this analysis to those from the melanogaster group. This set of species also represents the likely 
extent of major SP/SPR-mediated post-mating responses, as only these species express SPR at

high levels in the female reproductive tract and respond to injection of synthetic SP (Tsuda et al., 2015). Thus, our analyses identify proteins that might have evolved adaptively to further improve/refine network function in the past $\sim 15$ million years (Seetharam \& Stuart, 2013). The results of the sites analyses are shown in Table 1. Four proteins - CG9997, fra mauro, CG1652 and hadley - show significant evidence for having a class of amino acid sites that have evolved under recurrent positive selection across the melanogaster group of species. Three other proteins (antares, intrepid and CG17575) each have a class of sites found to be under

357 positive selection in the Model M7/M8 comparison, but these results are no longer significant when comparing Models M8 and M8a, suggesting that the class of more quickly evolving sites identified for each protein in Model M8 may be evolving neutrally rather than under positive selection.

While all members of a protein network have a degree of functional co-dependence, the male-expressed network proteins that have evolved adaptively are known to have specific, genetic interdependencies. CG9997, a serine protease homolog predicted to be catalytically

364 inactive, must be produced in the male accessory glands for CG1652, a C-type lectin, to be transferred to mated females (Ravi Ram \& Wolfner, 2009). Likewise, in the absence of CG1652,

366 CG9997 is not efficiently "processed" from its $45-\mathrm{kDa}$ form to its $36-\mathrm{kDa}$ form in mated females

367 (Ravi Ram \& Wolfner, 2009, Singh et al., 2018). The loss of either protein prevents SP from

368 accumulating on stored sperm in females. Recent work has shown that both CG9997 and

369 CG1652 also bind to sperm, though their sperm-binding is detectable only in the hours after

370 mating, while SP binding lasts for several days (Peng et al., 2005, Singh et al., 2018). CG9997

371 and CG1652 also show significant evidence of evolutionary rate covariation (Findlay et al., 
372 2014). These results suggest that pressure to maintain their functional interactions may be a

373 factor driving the adaptive evolution of CG9997 and CG1652, as has been observed for pairs of

374 interacting reproductive proteins in other systems (Clark et al., 2009, Grayson, 2015).

375 Other work on CG9997 is consistent with its adaptive evolution. Wong et al. (2008)

376 found evidence for recent positive selection acting on this gene by examining patterns of

377 polymorphism and divergence between populations of D. melanogaster and D. simulans. They

378 hypothesized that non-catalytically active serine protease homologs like CG9997 function as

379 agonists or antagonists for active proteases. Another possibility, which is not mutually

380 exclusive, is that protease homologs bind to other proteins or molecules in the female tract to

381 slow their rate of digestion by active, female-derived proteases (LaFlamme \& Wolfner, 2013).

382 Under either scenario, protease homologs like CG9997 may need to continually coevolve with

383 their interacting partners, providing the impetus for the recurrent, adaptive evolution detected

384 here. Additionally, knockdown of CG9997 diminishes male sperm competitive ability (Castillo

$385 \&$ Moyle, 2014), suggesting another potential factor in its adaptive evolution.

Less functional information exists for the adaptively evolving, female-expressed proteins.

387 Both fra mauro and hadley were identified in a screen for female-expressed proteins that

388 coevolved with a male-expressed SP network protein; in each case, the coevolutionary signal

389 was with CG17575 (Findlay et al., 2014). RNAi knockdown of either gene reduced female

390 fertility, though knockdown females could receive SP and store it properly on sperm (Findlay et

391 al., 2014). These data suggested that the proteins could be involved in maintaining the female

392 long-term response to SP, though fra mauro knockdown females also showed a significant

393 fertility defect in the $24 \mathrm{hrs}$ after mating (Findlay et al., 2014). The fra mauro protein encodes a

394 predicted neprilysin protease, which may coevolve with its as yet unknown molecular targets or 
antagonists (LaFlamme \& Wolfner, 2013). As noted above, functional domains have not been identified for the hadley protein, so it is difficult to speculate on potential forces driving its

397 adaptive evolution.

Notably, several proteins in the SP network showed no evidence of recurrent adaptive evolution within the melanogaster group, while others had subsets of sites with evolutionary rates that were elevated, but approximated neutrality. These data suggest that while some network proteins may contain regions that are under relaxed constraint, much of the functionality

402 and interdependence of the network might have already existed at the origin of the melanogaster 403 group.

405 Several network proteins underwent adaptive evolution on specific lineages correlating with changes in reproductive phenotypes

While the PAML sites test described above detects recurrent adaptive evolution, protein networks can also be shaped by bursts of episodic positive selection acting on specific

409 phylogenetic lineages. One important evolutionary transition for the SP network occurred at the

410 base of the melanogaster group, when $S P R$ evolved expression in the lower female reproductive

411 tract (Tsuda et al., 2015). This change likely created (or exacerbated) a selective pressure for

412 higher SP levels in this location, as prolonged SP-SPR signaling could promote continued egg

413 production and prolong female non-receptivity to re-mating. Because a primary purpose of the

414 male-expressed SP network proteins in D. melanogaster is to bind SP to sperm to prolong the

415 post-mating response, we hypothesized that some of these proteins might have experienced a

416 burst of adaptive evolution on the same phylogenetic branch on which female reproductive SPR

417 expression is inferred to have evolved. Likewise, the increase in $S P R$ expression in females 
418 could have created a selective pressure for other female-expressed members of the network to

419 evolve. To test these ideas, we used the Venkat model, a modified PAML branch-sites test

420 (Venkat et al., 2018, Zhang et al., 2005), to ask whether any network protein had a subset of sites

421 under selection on the branch leading to the melanogaster group (i.e., branch 11 in Fig. 1).

422 Table 2 (left columns) shows the results of these tests. Two proteins show marginal

423 evidence for adaptive evolution on branch 11, leading to the melanogaster group: CG1656 and

424 SPR. As originally formulated (Zhang et al., 2005), the LRT for the branch-sites test follows a

425 null distribution described as an equal mixture of point mass 0 and a chi-square distribution with

4261 degree of freedom (df). Under this null distribution, the test statistic corresponding with a $p$ -

427 value of 0.05 is 2.71, a value exceeded in tests of both CG1656 and SPR. However, the test is

428 typically conducted conservatively (Venkat et al., 2018, Zhang et al., 2005), following only a

429 chi-square distribution with 1 df. The p-values listed in Table 2 are calculated based on this

430 latter distribution, and they are marginally non-significant $(0.05<p<0.1)$ for CG1656 and SPR.

431 The potential adaptive evolution of sites in the SPR protein along branch 11 is

432 interesting, because this lineage also represents the time during which the protein became

433 expressed in the female reproductive tract (Tsuda et al., 2015). Thus, it is possible that the SPR

434 gene underwent both regulatory and protein-coding adaptations that altered how the female post-

435 mating response is controlled. The other protein that potentially underwent adaptive evolution

436 along this lineage is the predicted C-type lectin CG1656, which functions similarly to its

437 recurrently rapidly evolving paralog described above, CG1652. Both lectins are required for

438 SP's long-term binding to stored sperm, and both proteins themselves bind sperm temporarily in

439 the hours after mating (Singh et al., 2018). Given the potential selective pressure to bind more

440 SP to stored sperm in female tracts expressing $S P R$, it is possible that the adaptive evolution of 
441 CG1656 on this key phylogenetic branch could have helped to improve the efficiency of SP's

442 binding to sperm. This idea could be tested in future experiments by either identifying and

443 mutating the residues likely to have changed along branch 11 and/or by substituting an outgroup

444 ortholog of CG1656 (and potentially its duplicate, CG1652) into D. melanogaster and examining

445 the effects on SP's sperm binding and on the female long-term post-mating response.

446 Prior work demonstrated that SP binds to the full length of $D$. melanogaster sperm (Peng

447 et al., 2005, Ravi Ram \& Wolfner, 2009, Singh et al., 2018). Indeed, the ability of SP (and

448 potentially other molecules) to bind sperm and then influence post-mating responses is one

449 hypothesis for why sperm tails have evolved to be so long in many Drosophila species. Within

450 the melanogaster group species that experience SP-mediated post-mating responses, one notable

451 change in reproductive physiology is that the sperm of D. ananassae and its closely related

452 species are considerably longer than those of D. melanogaster (D. ananassae sperm length: 3.3

$453 \mathrm{~mm}$; D. melanogaster and other melanogaster group species sperm length: just under $2 \mathrm{~mm}$

454 (Pitnick et al., 1999, Joly \& Bressac, 1994, Markow, 1996)). We thus infer that a major $(>50 \%)$

455 increase in sperm length occurred on the branch of the phylogeny leading to D. ananassae and

456 D. bipectinata (branch 15 in Fig. 1).

457 To test for whether any SP network proteins experienced adaptive evolution concurrent

458 with this change in sperm length, we again used the modified branch-sites test. Two network

459 proteins, antares and CG17575, show evidence of positive selection acting on specific sites on

460 the lineage leading to D. ananassae and D. bipectinata (Table 2, right columns). Antares' signal

461 of selection is significant under both null distributions described above, while CG17575's signal

462 is significant under the mixed null distribution and approached significance $(p=0.0504)$ under

463 the conservative test. In addition to facilitating SP's long-term binding to sperm, antares also 
464 binds to sperm itself for a shorter period (Findlay et al., 2014, Singh et al., 2018). Thus, antares

465 might have evolved adaptively to facilitate greater or more efficient binding of either itself or SP

466 to sperm as sperm tails lengthened. Interestingly, the antares ortholog in outgroup species $D$.

467 mojavensis and D. arizonae was also found to evolve under diversifying selection (Bono et al.,

468 2015), even though D. mojavensis does not have a currently detectable SP ortholog (Tsuda et al.,

469 2015) (Fig. 2). Heterospecific matings between these species fail due to post-mating, pre-zygotic

470 isolating barriers, which include problems with sperm storage in the female reproductive tract

471 (Kelleher \& Markow, 2007). It is thus possible that antares plays an essential role in binding

472 molecules to sperm and/or facilitating sperm storage, and that the male reproductive activity of

473 antares has been refined by different selective pressures in different lineages.

474 CG17575 is a male-expressed, cysteine-rich secretory protein required for SP and other

475 sperm-binding network proteins to localize from the female uterus, where seminal proteins and

476 sperm are first deposited, into the seminal receptacle (SR), the primary site of sperm storage in

477 D. melanogaster (Ravi Ram \& Wolfner, 2009, Singh et al., 2018). Since CG17575 does not

478 itself bind sperm (Singh et al., 2018), further details of how CG17575 provides for proper

479 localization of other seminal proteins to the seminal receptacle are needed before we can

480 speculate on the selective forces that might have contributed to its evolution in this lineage.

481 The branch-sites tests for branches 11 and 15 reported above were conducted using full-

482 length gene sequences, since the test has limited power. However, we repeated this analysis on

483 all segments of the six genes for which recombination was detected. These results (Table S4)

484 found marginal evidence for selection for antares on branch 11 and for a segment of CG1652 on

485 branch 15. CG1656 was not among the genes for which recombination was detected (Table S2),

486 so its results above are unaltered. 
Seminase gene duplicates retain male-specific expression patterns across melanogaster group species

In addition to CG17575, the male-expressed serine protease seminase is required for the

491 localization of SP and other male-expressed proteins to the SR after mating (LaFlamme et al.,

492 2012, Singh et al., 2018). Seminase arose through gene duplication in the lineage leading to the

493 melanogaster group of flies. The genomes of D. pseudoobscura and other outgroup species

494 contain only one detectable copy of the gene, but in D. melanogaster and its fellow melanogaster

495 group members, there are three tandemly arrayed, intron-containing copies, suggesting two

496 distinct DNA-based duplication events. The other genes are CG10587 and CG11037. Like

497 seminase, both are expressed specifically in the male accessory gland in D. melanogaster

498 (Brown et al., 2014, Leader et al., 2018). While we detected no recurrent or episodic positive

499 selection acting on seminase after these duplications (Tables 1-2), we were curious whether it or

500 its paralogs might have evolved different expression patterns (and, thus, potential functions) after

501 duplication. We thus performed RT-PCR to amplify each paralog from cDNA isolated from

502 males or females of a variety of species from the melanogaster group. We also assessed the

503 expression of the single-copy parent gene from D. pseudoobscura and D. willistoni. Our results

504 (Figure 3) show that both the single-copy genes from the outgroup species, as well as all of

505 paralogs from all melanogaster group species tested, are expressed specifically in adult males.

506 This result is consistent with the ancestral single copy of seminase also functioning in male

507 reproduction (and potentially with other SP network proteins). 
510 to have unique roles, in spite of their conserved expression patterns. Future studies should

511 evaluate how the paralogs contribute to reproduction, which may suggest possible evolutionary

512 forces that affected their evolution after the gene duplication events. 


\section{Conclusions}

514 Sex peptide is directly responsible for major changes in female post-mating behavior and

515 physiology and is therefore one of the best characterized reproductive proteins to date. SP-

516 mediated responses appear to have arisen specifically in the melanogaster group of Drosophila,

517 and they manifest in full only with the help of a suite of male- and female-derived proteins, the

518 SP network. We have shown that these proteins are present and expressed in species outside of

519 the melanogaster group, suggesting they likely function in reproduction in these species and that

520 they did so in a common ancestor. Within the melanogaster group, several network proteins

521 (CG9997, CG1652, fra mauro, and hadley) have experienced recurrent positive selection,

522 suggesting that continued, adaptive evolution refined SP network function. A non-overlapping

523 set of proteins, including CG1656, SPR, antares, and CG17575, showed some evidence of bursts

524 of adaptive evolution on specific phylogenetic lineages corresponding with major changes in SP

525 network reproductive phenotypes. Taken together, these data suggest that SP network proteins

526 may have interacted to affect reproduction before the evolution of major SP-mediated changes in

527 the melanogaster group. However, once SPR became expressed at high levels in the female

528 reproductive tract in the common ancestor of this group (Tsuda et al., 2015), a combination of

529 both quick bursts of adaptation on specific lineages and recurrent changes at specific protein sites

530 helped the network evolve into the present form observed in D. melanogaster. This study

531 demonstrates how changes in both regulatory and protein-coding regions can affect the evolution

532 of protein networks and motivates future functional studies of the SP network proteins in

533 Drosophila species both within and outside of the melanogaster group. 


\section{Acknowledgements}

535 We thank L. Moyle, M. Hahn, A. Venkat and M. Wu for assistance in implementing the

536 Venkat model, D. Thakral for help developing batch PAML scripts, K. Ober and M. Wolfner for

537 helpful discussions, and two reviewers for valuable feedback on the manuscript. This work was

538 supported by NSF CAREER Award 1652013 and a summer research fellowship generously

539 provided by Dr. William Crowley. The authors report no conflicts of interest. 


\section{References}

Alpern, J. H. M., Asselin, M. M. \& Moehring, A. J. 2019. Identification of a novel sperm class and its role in fertilization in Drosophila. J Evol Biol 32: 259-266.

Apger-McGlaughon, J. \& Wolfner, M. F. 2013. Post-mating change in excretion by mated Drosophila melanogaster females is a long-term response that depends on sex peptide and sperm. J Insect Physiol 59: 1024-30.

Avila, F. W., Mattei, A. L. \& Wolfner, M. F. 2015. Sex peptide receptor is required for the release of stored sperm by mated Drosophila melanogaster females. Journal of Insect Physiology 76: 1-6.

Avila, F. W., Ravi Ram, K., Bloch Qazi, M. C. \& Wolfner, M. F. 2010. Sex peptide is required for the efficient release of stored sperm in mated Drosophila females. Genetics 186: 595600.

Bono, J. M., Matzkin, L. M., Hoang, K. \& Brandsmeier, L. 2015. Molecular evolution of candidate genes involved in post-mating-prezygotic reproductive isolation. J Evol Biol 28: 403-14.

Bono, J. M., Matzkin, L. M., Kelleher, E. S. \& Markow, T. A. 2011. Postmating transcriptional changes in reproductive tracts of con- and heterospecifically mated Drosophila mojavensis females. Proc Natl Acad Sci US A 108: 7878-83.

Brown, J. B., Boley, N., Eisman, R., May, G. E., Stoiber, M. H., Duff, M. O., Booth, B. W., Wen, J., Park, S., Suzuki, A. M., et al. 2014. Diversity and dynamics of the Drosophila transcriptome. Nature 512: 393-9.

Carvalho, G. B., Kapahi, P., Anderson, D. J. \& Benzer, S. 2006. Allocrine modulation of feeding behavior by the Sex Peptide of Drosophila. Curr Biol 16: 692-6.

Castillo, D. M. \& Moyle, L. C. 2014. Intraspecific sperm competition genes enforce post-mating species barriers in Drosophila. Proc Biol Sci 281: 20142050.

Chapman, T., Bangham, J., Vinti, G., Seifried, B., Lung, O., Wolfner, M. F., Smith, H. K. \& Partridge, L. 2003. The sex peptide of Drosophila melanogaster: female post-mating responses analyzed by using RNA interference. Proc Natl Acad Sci U S A 100: 9923-8.

Chen, P. S., Stumm-Zollinger, E., Aigaki, T., Balmer, J., Bienz, M. \& Bohlen, P. 1988. A male accessory gland peptide that regulates reproductive behavior of female D. melanogaster. Cell 54: 291-8.

Chen, Z. X., Sturgill, D., Qu, J., Jiang, H., Park, S., Boley, N., Suzuki, A. M., Fletcher, A. R., Plachetzki, D. C., FitzGerald, P. C., et al. 2014. Comparative validation of the D. melanogaster modENCODE transcriptome annotation. Genome Res 24: 1209-23.

Clark, N. L., Gasper, J., Sekino, M., Springer, S. A., Aquadro, C. F. \& Swanson, W. J. 2009. Coevolution of interacting fertilization proteins. PLoS Genetics 5: e1000570.

Drosophila 12 Genomes Consortium, Clark, A. G., Eisen, M. B., Smith, D. R., Bergman, C. M., Oliver, B., Markow, T. A., Kaufman, T. C., Kellis, M., Gelbart, W., et al. 2007. Evolution of genes and genomes on the Drosophila phylogeny. Nature 450: 203-18.

Felsenstein, J. (2005) PHYLIP (Phylogeny Inference Package) version 3.6. pp. Distributed by Author, Department of Genome Sciences, University of Washington, Seattle.

Findlay, G. D., Sitnik, J. L., Wang, W., Aquadro, C. F., Clark, N. L. \& Wolfner, M. F. 2014. Evolutionary Rate Covariation Identifies New Members of a Protein Network Required for Drosophila melanogaster Female Post-Mating Responses. PLoS Genetics 10: e1004108. 
Grayson, P. 2015. Izumo1 and Juno: the evolutionary origins and coevolution of essential spermegg binding partners. $R$ Soc Open Sci 2: 150296.

Gubala, A. M., Schmitz, J. F., Kearns, M. J., Vinh, T. T., Bornberg-Bauer, E., Wolfner, M. F. \& Findlay, G. D. 2017. The Goddard and Saturn Genes Are Essential for Drosophila Male Fertility and May Have Arisen De Novo. Mol Biol Evol 34: 1066-1082.

Hasemeyer, M., Yapici, N., Heberlein, U. \& Dickson, B. J. 2009. Sensory neurons in the Drosophila genital tract regulate female reproductive behavior. Neuron 61: 511-8.

Isaac, R. E., Li, C., Leedale, A. E. \& Shirras, A. D. 2010. Drosophila male sex peptide inhibits siesta sleep and promotes locomotor activity in the post-mated female. Proc Biol Sci 277: 65-70.

Jang, Y. H., Chae, H. S. \& Kim, Y. J. 2017. Female-specific myoinhibitory peptide neurons regulate mating receptivity in Drosophila melanogaster. Nat Commun 8: 1630.

Joly, D. \& Bressac, C. 1994. Sperm Length in Drososphilidae (Diptera): Estimation by Testis and Receptacle Lengths. International Journal of Insect Morphology \& Embryology 23: 85-92.

Kelleher, E. S. \& Markow, T. A. 2007. Reproductive tract interactions contribute to isolation in Drosophila. Fly (Austin) 1: 33-7.

Kelleher, E. S., Watts, T. D., LaFlamme, B. A., Haynes, P. A. \& Markow, T. A. 2009. Proteomic analysis of Drosophila mojavensis male accessory glands suggests novel classes of seminal fluid proteins. Insect Biochem Mol Biol 39: 366-71.

Kim, Y. J., Bartalska, K., Audsley, N., Yamanaka, N., Yapici, N., Lee, J. Y., Kim, Y. C., Markovic, M., Isaac, E., Tanaka, Y., et al. 2010. MIPs are ancestral ligands for the sex peptide receptor. Proc Natl Acad Sci U S A 107: 6520-5.

Kosakovsky Pond, S. L., Posada, D., Gravenor, M. B., Woelk, C. H. \& Frost, S. D. 2006. GARD: a genetic algorithm for recombination detection. Bioinformatics 22: 3096-8.

LaFlamme, B. A., Ravi Ram, K. \& Wolfner, M. F. 2012. The Drosophila melanogaster seminal fluid protease "Seminase" regulates proteolytic and post-mating reproductive processes. PLoS Genetics 8: 30-32.

LaFlamme, B. A. \& Wolfner, M. F. 2013. Identification and function of proteolysis regulators in seminal fluid. Mol Reprod Dev 80: 80-101.

Leader, D. P., Krause, S. A., Pandit, A., Davies, S. A. \& Dow, J. A. T. 2018. FlyAtlas 2: a new version of the Drosophila melanogaster expression atlas with RNA-Seq, miRNA-Seq and sex-specific data. Nucleic Acids Res 46: D809-D815.

Liu, H. \& Kubli, E. 2003. Sex-peptide is the molecular basis of the sperm effect in Drosophila melanogaster. Proc Natl Acad Sci U S A 100: 9929-33.

Madeira, F., Park, Y. M., Lee, J., Buso, N., Gur, T., Madhusoodanan, N., Basutkar, P., Tivey, A. R. N., Potter, S. C., Finn, R. D., et al. 2019. The EMBL-EBI search and sequence analysis tools APIs in 2019. Nucleic Acids Res 47: W636-W641.

Markow, T. A. (1996) Evolution of Drosophila mating systems. In: Evolutionary Biology, Vol. 29. pp. 73-106.

Markow, T. A. \& O'Grady, P. M. 2005. Evolutionary genetics of reproductive behavior in Drosophila: connecting the dots. Annual Review of Genetics 39: 263-291.

McDonough, C. E., Whittington, E., Pitnick, S. \& Dorus, S. 2016. Proteomics of reproductive systems: Towards a molecular understanding of postmating, prezygotic reproductive barriers. J Proteomics 135: 26-37. 
Oh, Y., Yoon, S. E., Zhang, Q., Chae, H. S., Daubnerova, I., Shafer, O. T., Choe, J. \& Kim, Y. J. 2014. A homeostatic sleep-stabilizing pathway in Drosophila composed of the sex peptide receptor and its ligand, the myoinhibitory peptide. PLoS Biol 12: e1001974.

Peng, J., Zipperlen, P. \& Kubli, E. 2005. Drosophila sex-peptide stimulates female innate immune system after mating via the toll and Imd pathways. Current Biology 15: 16901694.

Pitnick, S., Markow, T. A. \& Spicer, G. S. 1999. Evolution of Multiple Kinds of Female SpermStorage Organis in Drosophila. Evolution 53: 1804-1822.

Poels, J., Van Loy, T., Vandersmissen, H. P., Van Hiel, B., Van Soest, S., Nachman, R. J. \& Vanden Broeck, J. 2010. Myoinhibiting peptides are the ancestral ligands of the promiscuous Drosophila sex peptide receptor. Cell Mol Life Sci 67: 3511-22.

Ravi Ram, K. \& Wolfner, M. F. 2007. Sustained post-mating response in Drosophila melanogaster requires multiple seminal fluid proteins. PLoS Genetics 3: 2428-2438.

Ravi Ram, K. \& Wolfner, M. F. 2009. A network of interactions among seminal proteins underlies the long-term postmating response in Drosophila. Proceedings of the National Academy of Sciences of the United States of America 106: 15384-15389.

Rezaval, C., Pavlou, H. J., Dornan, A. J., Chan, Y. B., Kravitz, E. A. \& Goodwin, S. F. 2012. Neural circuitry underlying Drosophila female postmating behavioral responses. Curr Biol 22: 1155-65.

Schnakenberg, S. L., Matias, W. R. \& Siegal, M. L. 2011. Sperm-storage defects and live birth in Drosophila females lacking spermathecal secretory cells. PLoS Biol 9: e1001192.

Seetharam, A. S. \& Stuart, G. W. 2013. Whole genome phylogeny for 21 Drosophila species using predicted 2b-RAD fragments. PeerJ 1: e226.

Singh, A., Buehner, N. A., Lin, H., Baranowski, K. J., Findlay, G. D. \& Wolfner, M. F. 2018. Long-term interaction between Drosophila sperm and sex peptide is mediated by other seminal proteins that bind only transiently to sperm. Insect Biochem Mol Biol 102: 43-51.

Singh, S. R., Singh, B. N. \& Hoenigsberg, H. F. 2002. Female remating, sperm competition and sexual selection in Drosophila. Genetics and Molecular Research 1: 178-215.

Sitnik, J. L., Gligorov, D., Maeda, R. K., Karch, F. \& Wolfner, M. F. 2016. The Female PostMating Response Requires Genes Expressed in the Secondary Cells of the Male Accessory Gland in Drosophila melanogaster. Genetics 202: 1029-41.

Snook, R., Markow, T. \& Karr, T. 1994. Functional nonequivalence of sperm in Drosophila pseudoobscura. Proceedings of the National Academy of Sciences of the United States of America 91: 11222-11226.

Snook, R. R. 1995. The Evolution of Sperm Polymorphism in the Drosophila Obscura Group. 957.

Soller, M., Bownes, M. \& Kubli, E. 1999. Control of oocyte maturation in sexually mature Drosophila females. Dev Biol 208: 337-51.

Swanson, W. J., Nielsen, R. \& Yang, Q. 2003. Pervasive adaptive evolution in mammalian fertilization proteins. Molecular biology and evolution 20: 18-20.

Tamura, K., Stecher, G., Peterson, D., Filipski, A. \& Kumar, S. 2013. MEGA6: Molecular evolutionary genetics analysis version 6.0. Molecular Biology and Evolution 30: 27252729.

Tange, O. 2018. GNU Parallel 2018.

Tsuda, M., Peyre, J. B., Asano, T. \& Aigaki, T. 2015. Visualizing Molecular Functions and Cross-Species Activity of Sex-Peptide in Drosophila. Genetics: 1-10. 
Venkat, A., Hahn, M. W. \& Thornton, J. W. 2018. Multinucleotide mutations cause false inferences of lineage-specific positive selection. Nat Ecol Evol.

Weaver, S., Shank, S. D., Spielman, S. J., Li, M., Muse, S. V. \& Kosakovsky Pond, S. L. 2018. Datamonkey 2.0: a modern web application for characterizing selective and other evolutionary processes. Mol Biol Evol 35: 773-777.

Wilburn, D. B. \& Swanson, W. J. 2016. From molecules to mating: Rapid evolution and biochemical studies of reproductive proteins. J Proteomics 135: 12-25.

Wong, A., Turchin, M. C., Wolfner, M. F. \& Aquadro, C. F. 2008. Evidence for positive selection on Drosophila melanogaster seminal fluid protease homologs. Mol Biol Evol 25: 497-506.

Yamanaka, N., Hua, Y. J., Roller, L., Spalovska-Valachova, I., Mizoguchi, A., Kataoka, H. \& Tanaka, Y. 2010. Bombyx prothoracicostatic peptides activate the sex peptide receptor to regulate ecdysteroid biosynthesis. Proc Natl Acad Sci U S A 107: 2060-5.

Yang, C. H., Rumpf, S., Xiang, Y., Gordon, M. D., Song, W., Jan, L. Y. \& Jan, Y. N. 2009. Control of the postmating behavioral switch in Drosophila females by internal sensory neurons. Neuron 61: 519-26.

Yang, H., Jaime, M., Polihronakis, M., Kanegawa, K., Markow, T., Kaneshiro, K. \& Oliver, B. 2018. Re-annotation of eight Drosophila genomes. Life Sci Alliance 1: e201800156.

Yang, Z. 1998. Likelihood Ratio Tests for Detecting Positive Selection and Application to Primate Lysozyme Evolution. Molecular Biology and Evolution 15: 568-573.

Yang, Z. 2007. PAML 4: Phylogenetic analysis by maximum likelihood. Molecular Biology and Evolution 24: 1586-1591.

Yang, Z. \& Dos Reis, M. 2011. Statistical properties of the branch-site test of positive selection. Molecular Biology and Evolution 28: 1217-1228.

Yang, Z., Nielsen, R., Goldman, N. \& Pedersen, A.-M. K. 2000. Codon-substitution models to detect adaptive evolution that account for heterogeneous selective pressures among site classes. Molecular biology and evolution 19: 49-57.

Yapici, N., Kim, Y.-J., Ribeiro, C. \& Dickson, B. J. 2008. A receptor that mediates the postmating switch in Drosophila reproductive behaviour. Nature 451: 33-37.

Zhang, J., Nielsen, R. \& Yang, Z. 2005. Evaluation of an improved branch-site likelihood method for detecting positive selection at the molecular level. Molecular Biology and Evolution 22: 2472-2479. 


\section{$712 \quad$ Figure Legends}

713

714

715

716

717

718

719

720

721

722

723

724

725

726

727

728

729

730
Figure 1. Phylogeny of Drosophila species examined in this study. The gray box indicates the melanogaster group. Each branch is numbered for reference in the main text. Key changes in reproductive tracts are indicated by letters a-d and are based on an examination of the literature (references cited in main text). The PAML branch-sites tests (see Results) were conducted on branches 11 and 15. Branch lengths are not proportional to evolutionary distances.

Figure 2. Bioinformatic identification of SP network proteins across 22 Drosophila species. Identified orthologs that were also reciprocal best BLAST hits are noted with a + sign, while a sign indicates no ortholog could be identified.

Figure 3. RT-PCR on seminase and paralogs shows conserved, male-biased expression after duplication. Orthologs of seminase, CG10586 and CG11037 show male-specific expression in various melanogaster group species, though the level of expression between paralogs and species is somewhat variable. The single-copy parent gene in D. pseudoobscura and D. willistoni is also expressed in a male-specific manner. The non-reproductive housekeeping gene, $R p L 32$, was included as a positive control for successful cDNA synthesis from both sexes. 
Table 1. PAML sites tests for positive selection acting on SP network genes within the melanogaster group. Genes for which recombination was detected are split into numbered segments as indicated. Specific codons that were inferred to be under selection by PAML's

734 Bayes Empirical Bayes (BEB) analysis with $\operatorname{Pr}>0.9$ are shown for genes or segments for which

735

736 positive selection was detected (i.e., in which model M8 was a significantly better fit to the data than models M7 and M8a). Amino acid site positions and identities refer to the D. melanogaster protein sequence.

738

\begin{tabular}{|c|c|c|c|c|c|c|c|c|}
\hline \multirow{2}{*}{ Gene } & \multirow{2}{*}{$\begin{array}{c}\text { Segment } \\
\text { Sites }\end{array}$} & \multirow{2}{*}{$\begin{array}{c}\text { M0 } \omega \\
\text { estimate }\end{array}$} & \multicolumn{2}{|c|}{ M7 vs. M8 } & \multicolumn{2}{|c|}{ M8 vs. M8a } & \multirow{2}{*}{$\begin{array}{l}\% \text { sites } \\
\text { in } \omega>1\end{array}$} & \multirow{2}{*}{$\begin{array}{c}\text { Sites with BEB } \\
\operatorname{Pr}>\mathbf{0 . 9 0}\end{array}$} \\
\hline & & & $2 * \Delta \operatorname{lnL}$ & $p$-value & $2 * \Delta \ln L$ & $p$-value & & \\
\hline antr & & 0.22 & 10.194 & 0.0061 & 1.935 & 0.1643 & & \\
\hline aqrs & & 0.21 & 3.950 & 0.1388 & 0.773 & 0.3792 & & \\
\hline CG1652_1 & $1-70$ & 0.08 & 5.877 & 0.0529 & 0.797 & 0.3721 & & \\
\hline CG1652 2 & $71-106$ & 0.01 & 0 & 1.0000 & 0 & 1.0000 & & \\
\hline CG1652_3 & $107-144$ & 0.01 & 4.822 & 0.0897 & 0 & 1.0000 & & \\
\hline CG1652_4 & $145-187$ & 0.03 & 0 & 1.0000 & 0 & 1.0000 & & \\
\hline CG1652_5 & 188-322 & 0.67 & 6.953 & 0.0309 & 4.398 & 0.0360 & $38 \%$ & $\begin{array}{c}233 P, 234 G, \\
250 \mathrm{~V} \\
\end{array}$ \\
\hline CG1656_1 & $1-69$ & 0.19 & 0.816 & 0.6651 & 0 & 1.0000 & & \\
\hline CG1656_2 & $70-328$ & 0.08 & 2.981 & 0.2252 & 0 & 1.0000 & & \\
\hline CG9997 & & 0.25 & 21.001 & $2.75 \mathrm{E}-05$ & 5.974 & 0.0145 & $10 \%$ & $152 S$ \\
\hline CG17575_1 & $1-139$ & 0.03 & 0 & 1.0000 & 0 & 1.0000 & & \\
\hline CG17575_2 & $140-298$ & 0.08 & 16.612 & 0.0002 & 1.373 & 0.2413 & & \\
\hline Esp & & 0.03 & 0 & 1.0000 & 2.096 & 0.1477 & & \\
\hline frma_1 & 1-347 & 0.26 & 13.421 & 0.0012 & 4.515 & 0.0336 & $11 \%$ & none \\
\hline frma_2 & 348-611 & 0.21 & 8.188 & 0.0167 & 6.106 & 0.0135 & $2 \%$ & 392A \\
\hline hdly_1 & $1-364$ & 0.30 & 42.194 & 0.0000 & 37.383 & 0.0000 & $7 \%$ & $\begin{array}{c}\text { 173V, 201I, } \\
\text { 229S, 239I, } \\
\text { 304A }\end{array}$ \\
\hline hdly_2 & $365-445$ & 0.12 & 0.494 & 0.7813 & 0.463 & 0.4964 & & \\
\hline intr & & 0.21 & 6.719 & 0.0348 & 0.547 & 0.4597 & & \\
\hline sems_1 & 1-93 & 0.28 & 0.640 & 0.7262 & 0.409 & 0.5227 & & \\
\hline sems 2 & $94-275$ & 0.18 & 0.899 & 0.6379 & 0.320 & 0.5715 & & \\
\hline SP & & 0.22 & 1.463 & 0.4813 & 0 & 1.0000 & & \\
\hline SPR & & 0.04 & 1.817 & 0.4031 & 8.828 & 0.0030 & & \\
\hline
\end{tabular}


Table 2. Venkat model branch-sites tests for positive selection acting on specific sites of SP

742 network proteins on two specific lineages. $P$-values are calculated based on a $\chi_{1}^{2}$ distribution.

743 Asterisks indicate likelihood ratio test statistics that reach the $p<0.05$ significance threshold for

744 a null distribution derived from a 50:50 ratio of point mass 0 and the $\chi_{1}^{2}$ distribution.

745

\begin{tabular}{|c|c|c|c|c|c|c|}
\hline \multirow{2}{*}{ Gene } & \multicolumn{2}{|c|}{$\begin{array}{c}\text { Branch 11: to melanogaster group } \\
(S P R \text { expressed in female tract) }\end{array}$} & \multicolumn{2}{c|}{$\begin{array}{c}\text { Branch 15: to D. ananassae/bipictenata } \\
\text { (increased sperm length) }\end{array}$} \\
\cline { 2 - 7 } & $\begin{array}{c}\text { Whole-Gene } \\
\text { w estimate }\end{array}$ & $\mathbf{2} * \Delta \mathbf{l n L}$ & $\boldsymbol{p}$-value & $\begin{array}{c}\text { Whole-Gene } \\
\text { ( estimate }\end{array}$ & $\mathbf{2} * \Delta \mathbf{l n L}$ & $\boldsymbol{p}$-value \\
\hline antr & 298.77 & 2.464 & 0.116 & $\mathbf{6 . 1 9}$ & $\mathbf{3 . 9 4 8}$ & $\mathbf{0 . 0 4 7}$ \\
\hline aqrs & 1.00 & 0 & 1.000 & 2.53 & 0.388 & 0.533 \\
\hline CG1652 & 7.62 & 1.428 & 0.232 & 1.05 & 0.006 & 0.938 \\
\hline CG1656 & 122.47 & $3.238^{*}$ & 0.072 & 1.04 & 0 & 1.000 \\
\hline CG9997 & 1.04 & 0 & 1.000 & 1.05 & 0.022 & 0.882 \\
\hline CG17575 & 424.52 & 2.425 & 0.119 & 422.98 & $3.827 *$ & 0.050 \\
\hline Esp & 1.08 & 0 & 1.000 & 1.08 & 0 & 1.000 \\
\hline frma & 1.08 & 0.034 & 0.854 & 1.04 & 0.012 & 0.913 \\
\hline hdly & 1.08 & 0 & 1.000 & 1.64 & 0.008 & 0.929 \\
\hline intr & 1.00 & 0.039 & 0.843 & 1.02 & 0 & 1.000 \\
\hline sems & 1.00 & 0 & 1.000 & 403.51 & 0.962 & 0.327 \\
\hline SP & 1.00 & 0 & 1.000 & 1.04 & 0.002 & 0.963 \\
\hline SPR & 9999.99 & $3.064 *$ & 0.080 & 1.08 & 0 & 1.000 \\
\hline
\end{tabular}




\section{Supplemental Materials}

Table S1. Orthologs used for each gene in PAML analyses. Some orthologs that were identified in Table 2 were excluded from PAML analysis due to unresolved intron borders and/or poor alignment quality. Only species above the dotted line (the melanogaster group) were analyzed in the sites tests.

Table S2. GARD results showing inferred recombination breakpoints. Breakpoint positions refer to nucleotide positions in the alignment files used. However, since alignments include gaps, these positions do not necessarily have a 3:1 correspondence with the $D$. melanogaster amino acid positions reported in Tables 1-3. The first table shows recombination breakpoints detected for aligned sequences from the entire Drosophila genus, which were used for the branch and branch-sites tests. The second table shows recombination breakpoints detected for aligned sequences from only the melanogaster group, which were used for the sites tests.

\section{Figure S1. RNAseq data from $D$. pseudoobscura support reproductive functions for SP} network proteins in a species that lacks full-scale SP responses. A) D. pseudoobscura expression patterns for each member of the SP network. Dark shading indicates high expression levels, stripes indicate low (but detectable) expression, and no shading indicates no expression detected in a given sample. Male-derived network proteins show male-biased or male-specific expression, consistent with reproductive functions. B) Examples of D. pseudoobscura expression data for several SP network genes; shading in part (A) is based on these data. The RNAseq data were accessed via FlyBase and generated by Yang et al. (Yang et al., 2018).

Table S3. Branch tests for rate heterogeneity. Partitions were implemented in PAML analyses if they were significant in both the LH and RH tests. SP network proteins detected by GARD to have multiple recombination segments. The table shows results for both branch 11 and branch 15 tests. Only the six genes for which recombination was detected in the relevant trees are shown in the table. $P$-values are calculated based on a $\chi_{1}^{2}$ distribution. Asterisks indicate likelihood ratio test statistics that reach the $p<$ 0.05 significance threshold for a null distribution derived from a 50:50 ratio of point mass 0 and the $\chi_{1}^{2}$ distribution. 
Fig. 1

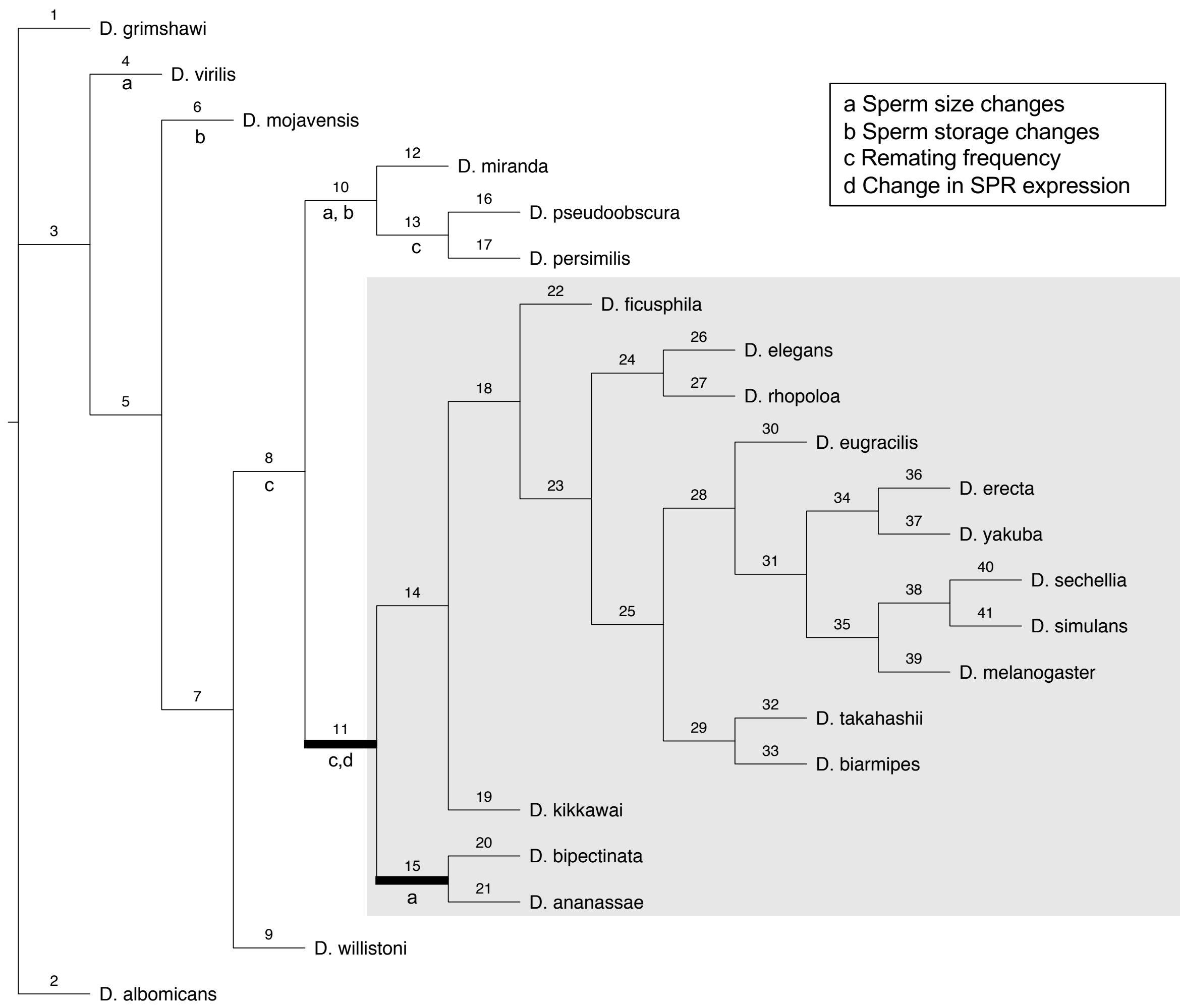




\begin{tabular}{|c|c|c|c|c|c|c|c|c|c|c|c|c|c|c|}
\hline \multirow[t]{23}{*}{ Fig. 2} & & intrepid & CG1652 & CG1656 & aquarius & antares & CG9997 & CG17575 & seminase & sex peptide & fra mauro & hadley & Esp & SPR \\
\hline & D.melanogaster & + & + & + & + & + & + & + & + & + & + & + & + & + \\
\hline & D.sechellia & + & + & + & + & + & + & + & + & + & + & + & + & + \\
\hline & D.simulans & + & + & + & + & + & + & + & + & + & + & + & + & + \\
\hline & D.erecta & + & + & + & + & + & + & + & + & + & + & + & + & + \\
\hline & D.yakuba & + & + & + & + & + & + & + & + & + & + & + & + & + \\
\hline & D.eugracilis & + & + & + & + & + & + & + & + & + & + & + & + & + \\
\hline & D.takahashii & + & + & + & + & + & + & + & + & + & + & + & + & + \\
\hline & D.biarmipes & + & + & + & + & + & + & + & + & + & + & + & + & + \\
\hline & D.elegans & + & + & + & + & + & + & + & + & + & + & + & + & + \\
\hline & D.rhopoloa & + & + & + & + & + & + & + & + & + & + & + & + & + \\
\hline & D.ficusphila & - & + & + & - & + & + & + & + & + & + & + & + & + \\
\hline & D.kikkawai & + & + & + & + & + & + & + & - & + & + & + & + & + \\
\hline & D.bipectinata & + & + & + & + & + & + & + & + & + & + & + & + & + \\
\hline & D.ananassae & + & + & + & + & + & + & + & + & + & + & + & + & + \\
\hline & D.pseudoobscura & + & + & + & + & + & + & + & + & + & + & + & + & + \\
\hline & D.persimilis & + & + & + & + & + & + & + & + & + & + & + & + & + \\
\hline & D.miranda & + & + & + & + & + & + & + & + & + & + & + & + & + \\
\hline & D. willistoni & + & + & + & + & + & + & + & + & + & + & + & + & + \\
\hline & D.mojavensis & - & + & + & + & + & + & + & + & - & + & + & + & + \\
\hline & D.virilis & + & + & + & + & + & + & + & + & + & + & + & - & + \\
\hline & D.albomicans & - & - & - & - & - & - & - & + & - & - & - & - & - \\
\hline & D.arimshawi & - & - & - & - & - & - & - & + & - & + & + & - & + \\
\hline
\end{tabular}


Fig. 3

\begin{tabular}{|c|c|c|c|c|}
\hline & seminase & CG11037 & CG10587 & $R p L 32$ \\
\hline & $M \quad F \quad g(-)$ & $M \quad F \quad g \quad(-)$ & $M \quad F \quad g \quad(-)$ & $M F g(-)$ \\
\hline D. melanogaster & 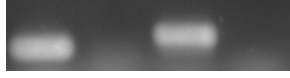 & 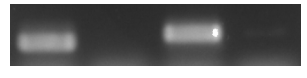 & $=$ & 50 \\
\hline D. yakuba & $\sigma$ & - & - & 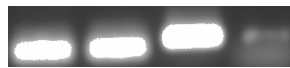 \\
\hline D. ficusphila & - & $?$ & - & \\
\hline D. bipectinata & {[} & 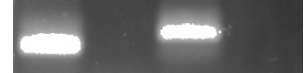 & 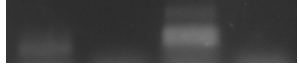 & $-8=$ \\
\hline D. ananassae & $\infty$ & $\Rightarrow$ & $-a$ & -20 \\
\hline D. pseudoobscura & & $-\infty$ & & $-\infty$ \\
\hline D. willistoni & & 0 & & $1 \mathbf{1}$ \\
\hline
\end{tabular}

